\title{
Work in Progress
}

\section{Screening of High Risk Infants for Metabolic Disease in a Metropolitan Hospital}

\author{
I. E. KRIEGER \\ Children's Hospital of Michigan, 3901 Beaubien, Detroit, MI 48201, USA \\ M. Nigro, A. SARnalk and Q. TAQI \\ Wayne State University. School of Medicine, Departments of Pediatrics and Neurology, \\ Children's Hospital of Michigan, Detroit, Michigan 48201, USA
}

Screening of symptomatic infants for metabolic diseases is described which led to an improved detection rate because of better awareness and case selection, as well as the use of a test for urinary organic acids.

We observed a marked increase during the past 2 years in the number of metabolic diseases detected at the Children's Hospital of Michigan, which is a 315-bed hospital with 14000 admissions per year. The reasons were therefore explored.

\section{METHODS}

Until 1978 screening of symptomatic patients for metabolic diseases consisted of a number of simple chemical urine tests (MET) (Thomas and Howell, 1973) and amino acid paper chromatography (AA) (Mabry and Karan, 1964; Mabry and Todd, 1963). A gas chromatographic urine organic acid (OA) screen was then added (Schlenk and Gellerman, 1960; Najamura et al., 1976), inclusion of tests for ammonia $\left(\mathrm{NH}_{4}\right)$, lactate $(\mathrm{LA})$ and pyruvate (PA) was recommended, and criteria for selective screening were developed.

\section{RESULTS}

With the joint MET and AA screen we performed 321 tests per year in 1978-9 and in the preceding 6 years 194 tests per year. We detected four disorders per year, or one per 80 tests, compared to one per year and a detection rate of one per 194 tests before. During the same 2 years 105 patients had urine OA screens. Four cases of methylmalonic acidaemia (MMAemia) and one glutaric acidaemia were diagnosed, giving a detection rate of one per 21 tests. In the preceding 6 years only one organic acidaemia was recognized. The high incidence of MMAemia in Michigan (five in 2 years, including one referral) was not due to unusual ethnic or family distribution. One-hundred and twenty-eight patients per year had tests of LA or LA and PA, and we established a positive diagnosis in two patients. No hyperammonaemia was detected. OA thus added 2.5 cases per year to the yield of the MET and AA screen and LA, PA and $\mathrm{NH}_{4}$ added one more per year.

The following disorders were diagnosed: two nonketotic hyperglycinaemias (McKusick 23830), one maple sugar urine disease (McKusick 24860), one argininosuccinic acidaemia (McKusick 20790), one tyrosinaemia

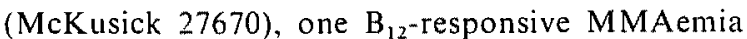
(McKusick 25110), and three $B_{12}$-unresponsive MMAemias (McKusick, 25100), one glutaric acidaemia (McKusick 23167), two fructose intolerances (McKusick 22960 ), two pyruvate dehydrogenase deficiencies (McKusick 20880), and one Sanfilippo mucopolysaccharidosis (McKusick 25290); total: 15 cases.

Unexplained catastrophic illness, episodic vomiting and/or acidosis and CNS depression were the presenting symptoms in 12 of 15 cases. A majority $(8 / 15)$ had a clinical course characterized by intermittent illness requiring intensive care. Consequently, 10 of 15 cases were recognized in an intensive care unit, compared to two of eight in the preceding years. Customary methods for mass screening of newborns would not have detected 10 of the 15 cases, because the disease was not associated with urine abnormality or the episodic nature caused abnormality only on occasions. Specific dietary or vitamin treatment was available in seven of 15 cases and five were treated successfully because of timely detection through screening.

\section{CONCLUSION}

As a result of intensified resident teaching, 1.63 times as many requests per year for routine metabolic screening tests (MET and AA) were received during 1978-9, and four times as many disorders were detected than before. We attribute this to:

(1) greater awareness by the staff of metabolic disease in general;

(2) better selection of symptomatic cases for screening, evidenced by an improved detection rate per test;

(3) more screening in intensive care units where $66 \%$ of the cases were detected, compared to $25 \%$ before.

Urine screening for $\mathrm{OA}$ by gas chromatography was partially responsible for the improved detection rate. Since four of the OAemias could have been detected by a simple chemical MMA screening test (Giorgio and Plant, 1965), routine use of such a test is recommended for screening of high risk infants. 


\section{References}

Giorgio, A. J. and Plant, G. W. E. A method for the colorimetric determination of urinary methylmalonic acid in pernicious anemia. J. Lab. Clin. Med.66 (1965) 667

Mabry, C. C. and Karan, E. A. Measurement of free amino acids in plasma and serum by means of high voltage paper electrophoresis. Am. J. Clin. Pathol. 34 (1964) 421

Mabry, C. C. and Todd, W. R. Quantitative measurement of individual and total free amino acids in urine. Rapid method employing high voltage paper electrophoresis and direct densitometry and its application to the urinary excretions of amino acids in normal subjects. J. Lab. Clin. Med.61 (196.3) 146
Najamura, E., Rosenberg, L. E. and Tanaka, K. Microdetermination of methylmalonic and other short chain dicarboxylic acids by gas chromatography. Clin. Chim. Acta $68(1976) 127$

Schlenk, H. and Gellerman, J. L. Esterification of fatty acids with diazomethane on a small scale. Anal. Chem. 32 (1960) 1412

Thomas, G. H. and Howell, R. R. Selected Screening Tests for Metabolic Diseases. Yearbook Medical Publishers, Inc., Chicago, 1973 